\title{
Which jet launching mechanism(s) in T Tauri stars?
}

\author{
J. Ferreira ${ }^{1}$, C. Dougados ${ }^{1}$, and S. Cabrit ${ }^{2}$ \\ 1 Laboratoire d'Astrophysique de Grenoble, BP 53, 38041 Grenoble Cedex, France \\ e-mail: Jonathan.Ferreira@obs.ujf-grenoble.fr \\ 2 Observatoire de Paris, LERMA, UMR 8112 du CNRS, 61 avenue de l'Observatoire, 75014 Paris, France
}

Received 20 September 2005 / Accepted 24 March 2006

\begin{abstract}
Aims. We examine whether ejection phenomena from accreting T Tauri stars can be described by only one type of self-collimated jet model.

Methods. We present analytical kinematic predictions valid soon after the Alfvén surface for all types of steady magnetically selfconfined jets.

Results. We show that extended disc winds, X-winds, and stellar winds occupy distinct regions in the poloidal speed vs. specific angular momentum plane. Comparisons with current observations of T Tauri jets yield quantitative constraints on the range of launching radii, magnetic lever arms, and specific energy input in disc and stellar winds. Implications on the origin of jet asymmetries and disc magnetic fields are outlined.

Conclusions. We argue that ejection phenomena from accreting T Tauri stars most likely include three dynamical components: (1) an outer self-collimated steady disc wind carrying most of the mass-flux in the optical jet (when present); confining (2) a pressure-driven coronal stellar wind; and (3) a hot inner flow made of blobs sporadically ejected from the magnetopause. If the stellar magnetic moment is parallel to the disc magnetic field, then the highly variable inner flow resembles a "Reconnection X-wind", that has been proven to efficiently brake down an accreting and contracting young star. If the magnetic moment is anti-parallel, then larger versions of the solar coronal mass ejections are likely to occur. The relative importance of these three components in the observed outflows and the range of radii involved in the disc wind are expected to vary with time, from the stage of embedded source to the optically revealed $\mathrm{T}$ Tauri star phase.
\end{abstract}

Key words. accretion, accretion disks - magnetohydrodynamics (MHD) - stars: pre-main sequence - ISM: jets and outflows

\section{Introduction}

Actively accreting "classical" T Tauri stars (TTS) often display supersonic collimated jets on scales of a few 10-100 AU in low excitation optical forbidden lines of [O I], [S II], and [N II] (Solf \& Boehm 1993; Hirth et al. 1994, 1997; Lavalley et al. 1997), with emission properties indicative of shock-excited outflowing gas at $\simeq 10^{4} \mathrm{~K}$ (Bacciotti \& Eislöffel 1999; Lavalley-Fouquet et al. 2000). These jet signatures are correlated with the infrared excess and accretion rate of the circumstellar disc (Cabrit et al. 1990; Hartigan et al. 1995). It is therefore widely believed that the accretion process is essential to the observed jets, although the precise physical connexion remains a matter of debate.

Over the last decade, new clues to the origin of the lowexcitation jets in TTS have been provided by sub-arcsecond observations of their collimation and kinematic properties, using HST or ground-based adaptive optics. A first important constraint is set by the narrow opening angles of a few degrees observed beyond $\simeq 50$ AU of the source (Burrows et al. 1996; Ray et al. 1996; Dougados et al. 2000; Hartigan et al. 2004). Since TTS do not possess dense envelopes that could confine the flow, the jets must be intrinsically collimated. To date, the only physical process capable of producing such unidirectional supersonic flows on the required scales is magnetohydrodynamic (MHD) self-confinement. This is achieved by the hoop stress due to a large scale open magnetic field anchored onto a rotating object (see reviews by Königl \& Pudritz 2000; Ferreira 2002). However, it remains to be established whether this MHD launching occurs predominantly from the circumstellar accretion disc, the rotating star, or its magnetosphere (or a combination of the above).

Interestingly, while $\mathrm{T}$ Tauri stars as a group have their discs oriented randomly with respect to the local magnetic field, those with bright optical jets tend to have their disc axes parallel to the ambient magnetic field direction (Ménard \& Duchêne 2004). This trend suggests that magnetic flux through the disc is a key parameter for the efficiency of collimated jets in TTS, as would be expected for if jets trace predominantly a disc wind ${ }^{1}$.

Recently, it has been realized that $\mathrm{T}$ Tauri jet kinematics offer a powerful way to constrain disc wind physics. Garcia et al. (2001) showed that classical "cold" self-similar disc wind solutions have excessive terminal speeds compared to forbidden line profiles in TTS jets, and are thus excluded, while the denser and slower "warm" disc winds are favored. Anderson et al. (2003) further demonstrated that the launching radius of a Keplerian disc wind may be simply derived from the jet rotation and poloidal speeds once the stellar mass is known, regardless of the details of the MHD disc wind solution. Interpreting the transverse velocity shifts measured by HST at the outer edges of three T Tauri jets as pure rotation motions, launching radii of 0.2 to 3 AU were inferred (Bacciotti et al. 2002; Anderson et al. 2003; Coffey et al. 2004), suggesting that at least the outer portions

1 The term "disc wind" is sometimes used in the literature to refer to a thermally-driven wind, namely an uncollimated slow outflow evaporating from the disc surface. In this paper, disc wind refers to a magnetically driven jet launched from the disc. 
(a)

Extended disc-wind: $r_{\mathrm{e}}>>\mathrm{r}_{\mathrm{i}}$

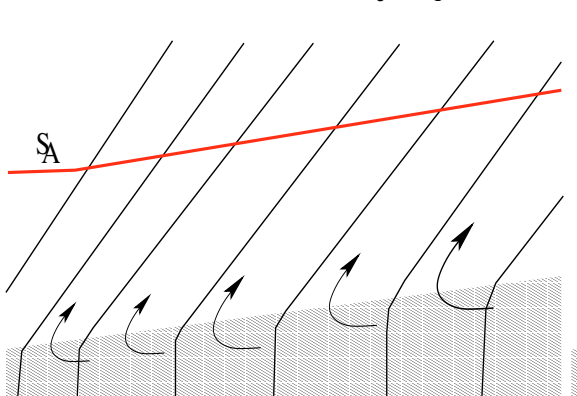

(d)

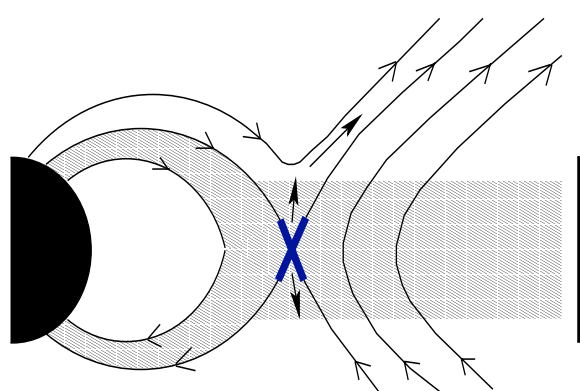

(b)

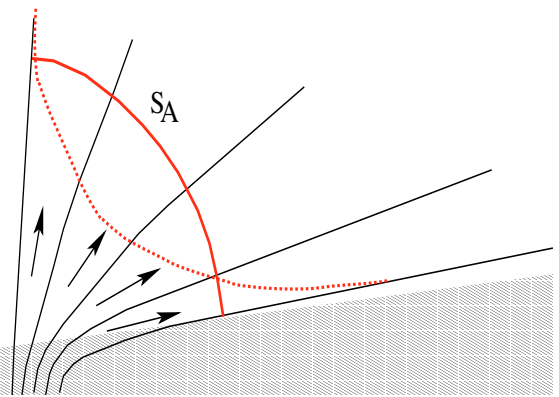

(e)

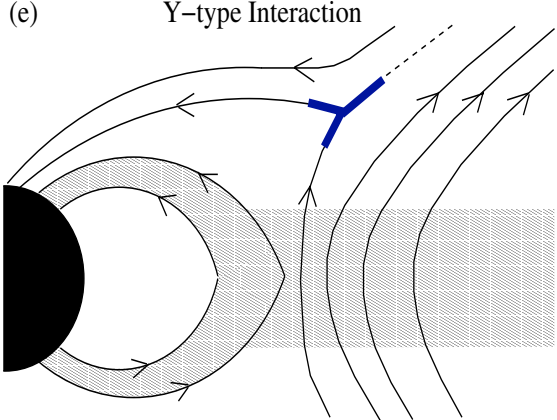

(c)

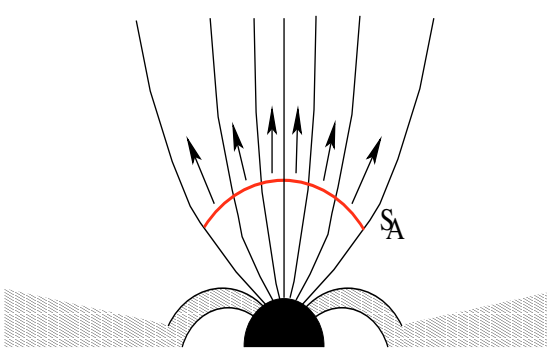

(f)

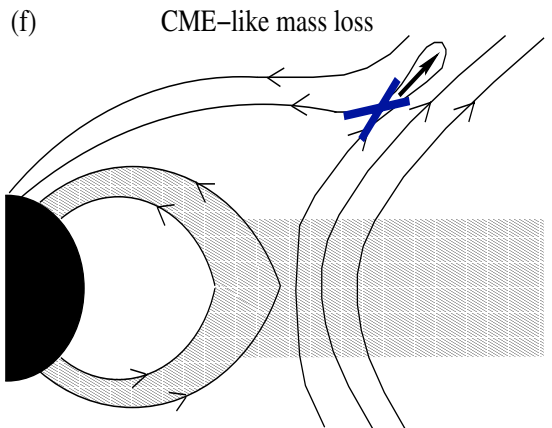

Fig. 1. Top: classes of published stationary MHD jets for YSOs. When the magnetic field is threading the disc on a large radial extension (a) extended disc wind) or a small disc annulus (b) X-wind), jets are accretion-powered. They are mostly pressure-driven when the field lines are anchored onto a slowly rotating star (c) stellar wind). The corresponding Alfvén surfaces $S_{\mathrm{A}}$ have been schematically drawn (thick lines). In the $\mathrm{X}$-wind case, two extreme shapes have been drawn: convex (solid line) and concave (dashed). Bottom: sketch of the two possible axisymmetric magnetospheric configurations: d) X-type neutral line driving unsteady Reconnection X-winds, when the stellar magnetic moment is parallel to the disc field; e) Y-type neutral line (akin the terrestrial magnetospheric current sheet) when the stellar magnetic moment is anti-parallel (or when the disc field is negligible). f) A CME-like ejection is produced whenever the magnetic shear becomes too strong in a magnetically dominated plasma. Such a violently relaxing event may occur with any kind of anti-parallel magnetospheric interaction (even with an inclined dipole). The thick lines mark the zones where reconnections occur.

of TTS optical jets would originate from extended regions of the disc surface. The small jet rotation speeds again rule out "cold" disc winds and favor "warm" disc wind solutions (Pesenti et al. 2004). These results raise several important questions:

- Could the same extended disc winds also explain the highvelocity component (HVC) at $200-400 \mathrm{~km} \mathrm{~s}^{-1}$ observed in forbidden lines closer to the jet axis (e.g. Bacciotti et al. 2000), or is another ejection process necessary? For example, Anderson et al. (2003) proposed that, while the rotating outer portions of the DG Tau jet (with flow speeds $\simeq 45 \mathrm{~km} \mathrm{~s}^{-1}$ ) trace a disc wind launched at $3 \mathrm{AU}$, the HVC would originate from a separate "X-wind" launched at the disc inner edge. Could these two types of disc winds really coexist?

- Is the current precision on rotation velocities in jets sufficient to rule out a dominant contribution from an X-wind, stellar wind, or magnetospheric wind to the observed optical jets? Are there further diagnostics that would allow to distinguish between these various scenarii?

The present paper is meant to address these issues. In Sect. 2, we recall the main physical ingredients of MHD ejection from the three sites that have been proposed to contribute to optical jets (disc, star, magnetosphere), discuss their collimation properties, and possible coexistence. In Sect. 3, we generalize the work of Anderson et al. (2003) by presenting analytical expressions for the poloidal and rotation speeds valid for all classes of steady, self-collimated MHD jets. We present a diagnostic diagram in the poloidal speed vs. specific angular momentum $\left(v_{\mathrm{p}}\right.$, $r v_{\phi}$ ) plane, valid soon after the Alfvén surface, and show that stellar winds, $\mathrm{X}$-winds and extended disc winds follow a distinct well-defined behavior. In Sect. 4, we place in this theoretical diagram the current observations of poloidal and rotation speeds in T Tauri jets, and discuss inferred constraints on model parameters (launching radii, magnetic lever arm, specific heat input). Section 5 summarizes our conclusions and their implications.

\section{MHD ejection from YSOs}

Figure 1 provides a synthetic illustration of the various configurations possibly leading to ejection in young stars. The top row displays steady ejection processes occurring regardless of the interaction between the star and its circumstellar disc: accretionpowered disc winds (Figs. 1a, b) and pure stellar winds (Fig. 1c). The bottom row displays the simplest possible magnetospheric star-disc interaction, namely a dipolar magnetic field aligned parallel (Fig. 1d) or anti-parallel (Figs. 1e,f) to the disc magnetic field. We detail below the properties and main physical ingredients of each ejection configuration.

\subsection{Accretion-powered disc winds}

In such models a large scale magnetic field is assumed to thread the disc from its inner radius $r_{\mathrm{i}} \sim r_{\mathrm{m}}$ (the magnetopause) to some external radius $r_{\mathrm{e}}$ (smaller than the outer disc radius). When $r_{\mathrm{e}} \gg$ $r_{\mathrm{i}}$ one gets a conventional "extended disc wind" as sketched in Fig. 1a, for which various self-similar solutions have been computed (Blandford \& Payne 1982; Wardle \& Königl 1993; Ferreira \& Pelletier 1995; Casse \& Ferreira 2000a; Ferreira \& Casse 2004). Figure 1b shows the other extreme situation when 
$r_{\mathrm{e}} \simeq r_{\mathrm{i}}$, namely when ejection occurs only from one annulus. This gives rise to a wide-angle "X-wind" (Shu et al. 1994; Shang et al. 2002).

Note that the only difference between these two cases is the amount of magnetic flux threading the disc. Actually, they correspond to two distinct scenarii for the origin of the large scale vertical magnetic field. In the "extended disc wind" paradigm, the field is assumed to have been either generated by dynamo or advected along with the accreting material (first in the infalling stage, then in the disc stage), or both. In the X-wind paradigm the disc is assumed to be devoid of any large scale magnetic field. It is further assumed that at some point in the past the stellar magnetospheric field, which is penetrating the inner disc regions, was flung open by outflowing disc material and that this has led to the actual field topology where there is no causal link anymore between the star and the disc. Despite this difference in the origin of the magnetic field, the ejection processes actually computed are identical. In both situations jets carry away the exact amount of angular momentum required to allow accretion in the underlying disc portion ${ }^{2}$. However, since the range in launching radii and the shape of the Alfvén surface are different, predicted terminal velocities and angular momentum fluxes are also different and can be tested against observations (see Sect. 3).

A common misconception in the literature is that "extended disc winds" would provide only low velocities and almost no collimation. For example, the MHD model of Wardle \& Königl (1993) matched a protostellar disc at 100 AU to a Blandford \& Payne (1982) jet model, which provided a confinement operating only at very large scales and low terminal speeds. However, this is not necessarily so. As shown by Cabrit et al. (1999) and Garcia et al. (2001), full MHD solutions including the disc wind transition (Ferreira 1997) reproduce very well the observed collimation of TTS jets and give adequate - or even excessive terminal speeds, provided their innermost launching radius $r_{\mathrm{i}}$ is $\simeq 0.07 \mathrm{AU}$ (typical disc corotation radius).

Another issue is whether an extended disc wind and an $\mathrm{X}$-wind may physically coexist. It has been proposed by Anderson et al. (2003) that an X-wind would be responsible for the HVC observed in the DG Tau jet whereas an extended disc wind, settled at 2-3 AU, would provide the slower gas flowing at the outer jet edges. This proposal raises the issue of the (vertical) magnetic field distribution within the disc. Indeed, it would imply that, say, from the inner disc edge at $\simeq 0.07$ AU (locus of the $\mathrm{X}$-wind) to 2-3 AU no large scale magnetic field is present (otherwise it would drive a disc wind). It is unclear how such a "hole" in the magnetic flux distribution could be obtained and maintained. Besides, the collimation (hence acceleration) of the inner X-wind would be strongly influenced by the pressure provided by the outer disc wind, thereby modifying the results based on the current published material. Therefore, it appears very unlikely that an X-wind could coexist with an extended disc wind. However, either type of disc wind may coexist with a stellar wind, and/or with some type of magnetospheric ejection (see below).

\subsection{MHD stellar winds}

Self-collimated stellar winds are produced from open stellar magnetic field lines as sketched in Fig. 1c. Stars rotating near break-up can provide their rotational energy to magnetically accelerate stellar winds. But in the case of slowly rotating young

${ }_{2}$ This property establishes a tight relation between mass-flux and terminal speed, discussed in more detail in Sect. 3.2. stars, such as T Tauri stars, stellar winds can hardly tap the protostellar rotational energy and must be therefore essentially driven by their pressure gradient (see Sect. 3.3 for more details). Such a gradient stems either from thermal effects (thermally-driven winds, e.g. Sauty \& Tsinganos 1994; Sauty et al. 2002) or from turbulent Alfvén waves (wave-driven winds, e.g. Hartmann \& MacGregor 1980; DeCampli 1981; Hartmann et al. 1982).

In $\mathrm{T}$ Tauri stars, accreting material is now believed to be channelled by the magnetospheric field and to release its mechanical energy at a high latitude shock, seen mostly as UV radiation. Under certain circumstances, some post-shocked material might keep a high temperature and build up a large enthalpy reservoir, allowing thereby for a thermally driven outflow. The magnetospheric accretion shock is also an efficient way to generate turbulent Alfvén waves. Thus, both thermal and turbulent pressures could well be present and help to launch MHD winds from the stellar surface. This would be expected to produce a correlation between accretion and ejection signatures, leading to "accretion-powered stellar winds" (Matt \& Pudritz 2005).

There is indeed mounting evidence for accretion-related, hot stellar winds in T Tauri stars e.g. in the form of broad blueshifted absorptions in high excitation lines (Edwards et al. 2003; Dupree et al. 2005), although their relation to the low-excitation large scale jets is unclear (Beristain et al. 2001). We thus make the conjecture that pressure-driven stellar winds likely represent one (fast) component of observed jets, filling in their innermost part. However, we will show in Sect. 4.4 that such stellar winds are unlikely to carry most of the jet mass-flux, as it would require an extremely efficient conversion of thermal/wave energy into kinetic energy along the jet.

\subsection{Unsteady magnetospheric winds}

The magnetospheric interaction between the protostar and its accretion disc provides several other possible driving mechanisms for outflows. However, in contrast to the previous situations, these outflows are intrinsically unsteady even in the simplified axisymmetric dipolar magnetic topology considered here.

\subsubsection{Parallel configuration: "ReX-winds"}

Let us assume that a large scale vertical magnetic field is present in the disc, allowing to drive an extended disc wind. If the protostellar magnetic moment is parallel to the disc field, then a magnetic neutral line (i.e. a true magnetic X-point in the meridional plane) forms at the disc midplane (Fig. 1d) and "Reconnection X-winds" (hereafter "ReX-winds") can be produced above this reconnection site (Ferreira et al. 2000). Accreted mass is lifted vertically above the neutral line by the strong Lorentz force and is loaded onto newly opened field lines. By this process, open field lines carried in by the accretion flow reconnect with closed stellar field lines. Therefore, ejected disc material is loaded onto field lines that are now anchored to the rotating protostar. Ejection occurs whenever the star rotates faster than the disc material, namely when the magnetic neutral line is located farther than the corotation radius. Both energy and angular momentum are thereby extracted from the rotating star and carried away in this outflow. Ferreira et al. (2000) showed that such a configuration provides a very efficient spin down of a contracting protostar, much more so than any of the other MHD ejection processes considered here.

Because of the intermittent nature of the mass loading process, ReX-winds are best seen as a series of bullets than a 
laminar flow. These bullets flow along (and push against) the inner magnetic surface of the disc wind. This is a very interesting situation for two reasons. First, the outer disc wind can provide also some confinement to this inner ReX-wind. Second, the presence of a high variability will probably lead to dissipation via shocks (thus heating) and provide an inner pressure (against the outer disc wind). Numerical simulations are needed to investigate such a "two-flow" configuration (see however Hirose et al. 1997; Miller \& Stone 1997). Note that the ReX-wind, although ejected from nearly the same region as the X-wind (corotation), has very different characteristics, namely: (i) an intrinsically unsteady character; (ii) a strong braking effect on the accreting star; (iii) the need for a large magnetic flux in the disc.

\subsubsection{Anti-parallel configuration: "CME-like ejecta"}

If, on the contrary, the stellar magnetic moment is anti-parallel to the disc field then a magnetopause is formed with no magnetic $\mathrm{X}$-point within the disc. This situation holds in particular in the $\mathrm{X}$-wind scenario, where most of the field at the inner disc edge is of stellar origin. The interface between the two fields adopts a Y shape above (and below) the disc, with a neutral line all the way at high altitudes (Fig. 1e) $)^{3}$. Such an interface does not allow to drive steady self-collimated jets. But the presence of this neutral line is a formidable site for time dependent energetic events, i.e reconnections, as illustrated in Fig. 1f. Indeed, any loop of stellar magnetic field that threads the disc will be sheared by the differential rotation between the disc and the star. This increase of magnetic energy relaxes by provoking an inflation of the loop. If the differential rotation continues then the final stage is a violent reconnection which leads to the ejection of a plasmoid at roughly $45^{\circ}$ (Hayashi et al. 1996; Goodson et al. 1997; Matt et al. 2002). This process is somewhat related to coronal mass ejections (CMEs) from the Sun and is believed to explain the release of giant X-ray flares in YSOs (Grosso et al. 2004).

One word of caution however. This physical process has only been analytically proven in the framework of force-free fields, namely when plasma inertia has no dynamical effect (Aly 1991). As observed by Romanova et al. (2002), when plasma inertia is important (full MHD equations), this violent opening of the field lines is not obtained. Instead, the magnetic configuration relaxes by modifying the rotation of the disc thereby lowering the differential rotation. According to these authors, all previous numerical situations showing CME-like ejecta (e.g. Matt et al. 2002) were in this magnetically dominated limit. If this is true, then the mass flux carried by such outflows becomes an issue if they were to explain all of the mass-loss observed in T Tauri jets. In that respect, it is noteworthy that the mass-flux ejected through this process, as modelled by Matt et al. (2002), does not seem to be correlated with the disc density, unlike what would be needed to reproduce the accretion-ejection correlation in T Tauri stars.

Another issue is collimation: $45^{\circ}$ is comparable to the opening angles of $\mathrm{T}$ Tauri jets very near their base (Hartigan et al. 2004), but much larger than the opening angles of a few degrees measured beyond 30-50 AU of their source (Burrows et al. 1996; Ray et al. 1996; Dougados et al. 2000). Intrinsic

${ }^{3}$ We do not consider the case with no large scale vertical magnetic field in the disc. Indeed, in our opinion, it is unlikely that the central star has built up its own magnetic field from that of the parent molecular cloud without any field left in the circumstellar disc. However, configurations (e) and (f) shown in Fig. 1 would also be obtained from a pure stellar field interacting with the inner disc (see e.g. Fig. 3 in Ostriker \& Shu 1995). collimation of CME-like ejecta cannot be invoked: Each ejected plasmoid is made of plasma carrying its own electric currents. But because of Ohmic resistivity (ion-electron collisions), these currents fade away and one gets eventually unmagnetized warm gas ejected into the interstellar medium. Without a proper global electric circuit there is no possibility for these plasmoids to develop a self-confining hoop-stress. Therefore, if CME-like ejecta were to explain HST jets, they must be confined by an outer pressure operating on small scales $(<30 \mathrm{AU})$. A rough estimate shows that such a confinement cannot be provided by the thermal pressure of the interstellar medium: the medium is too cold and the density should be about $10^{3}$ times larger than that of the outflow.

Alternatively, CME-like ejecta could be confined by an external magnetic pressure. In this case $\rho_{\mathrm{j}} v_{\mathrm{j}}^{2} \sim B_{\mathrm{ext}}^{2} / \mu_{\mathrm{o}}$ must hold at the axial distance $r_{\mathrm{j}}$ where confinement occurs. Outflowing mass conservation can be written as $\dot{M}_{\mathrm{j}}=S_{\mathrm{j}} \rho_{\mathrm{j}} v_{\mathrm{j}}$ where $\rho_{\mathrm{j}}$ is the density of mass ejected with velocity $v_{\mathrm{j}}$ through a surface of area $S_{\mathrm{j}}$. Since CME-like outflows would be produced inside a quite narrow solid angle, one expects $S_{\mathrm{j}} \ll 2 \pi r_{\mathrm{j}}^{2}$. Gathering this together, one gets the following estimate of the required magnetic field at $r_{\mathrm{j}}$,

$$
\begin{aligned}
B_{\mathrm{ext}} \simeq 25 & \left(\frac{\dot{M}_{\mathrm{j}}}{10^{-9} M_{\odot} / \mathrm{yr}}\right)^{1 / 2}\left(\frac{v_{\mathrm{j}}}{300 \mathrm{~km} \mathrm{~s}^{-1}}\right)^{1 / 2} \\
& \times\left(\frac{r_{\mathrm{j}}}{30 \mathrm{AU}}\right)^{-1}\left(\frac{2 \pi r_{\mathrm{j}}^{2}}{10 S_{\mathrm{j}}}\right)^{1 / 2} \mathrm{mG}
\end{aligned}
$$

This is far above the interstellar magnetic field value and shows that the poloidal magnetic field required to confine CME-like ejecta must have been amplified. This can only be done if it has been advected along by the infalling material. It must therefore be anchored on the underlying Keplerian accretion disc. The possible presence of such a large scale vertical magnetic field as well as its influence on the disc dynamics (e.g. launching a disc wind) have to be addressed.

To summarize, CME-like ejection raises critical issues on the mass loss and collimation and it remains to be proven that it could account, alone, for observed jets from $\mathrm{T}$ Tauri stars. ReX-winds have more promising properties, but they are necessarily occurring concurrently with extended disc winds. Thus, although time-dependent magnetospheric ejection episodes (related to reconnection events) are most likely present in TTS, they may not dominate the observed jet flow.

We also note that an interesting variability process could occur if young stars sustain dynamos with global polarity inversion. In the presence of a large scale magnetic field threading the disc, one would indeed observe cycles of an X-type interaction with efficient $\mathrm{ReX}$-wind ejection and magnetic braking, alternating with a quiescent Y-type interaction and sporadic CME-like ejections. Timescales of 10-20 yr (López-Martín et al. 2003) between jet knots might reflect such magnetic cycles.

\section{Kinematics of stationary MHD jets}

On small scales ( $<200 \mathrm{AU})$, spatially resolved jets from T Tauri stars observed in forbidden lines may be considered as essentially steady. Indeed, even though knots are commonly observed, the derived time scales are longer than the dynamical time scales involved in the acceleration zone. Besides, inferred shock velocities are much smaller than jet velocities $(\Delta v / v \leq 25 \%$, Lavalley-Fouquet et al. 2000). Thus, steady-state models should 
catch their main features. For the reasons outlined in the previous section, we assume in the following that the mass loss in CME-like ejecta is negligible with respect to the steady flow components from the disc (extended or X-wind) and/or the star. The remaining question is then: which of the latter components dominates the jet mass flux?

To search for possible kinematic diagnostics distinguishing among X-wind, extended disc wind, and stellar wind, we present in this section analytical relations for the poloidal and toroidal velocities valid for all types of super-Alfvénic, stationary, axisymmetric, self-collimated MHD jets. This is an extension of the work of Anderson et al. (2003). Since all available models are governed by the same set of ideal MHD equations, their differences arise only from different boundary conditions.

\subsection{Governing dynamical equations}

The poloidal magnetic field writes $\boldsymbol{B}_{\mathrm{p}}=\left(\nabla a \times \boldsymbol{e}_{\phi}\right) / r$, where $a(r, z)=$ const. describes a surface of constant magnetic flux. An MHD jet is made of nested magnetic surfaces with several integrals of motion along each surface. Using the usual definitions ( $v_{\mathrm{p}}$ poloidal velocity, $\Omega$ angular velocity and $\rho$ density), these are: (1) the mass to magnetic flux ratio $\eta(a)$ with $\boldsymbol{v}_{\mathrm{p}}=\eta(a) \boldsymbol{B}_{\mathrm{p}} / \mu_{0} \rho ;(2)$ the angular velocity of a magnetic surface $\Omega_{*}(a)=\Omega-\eta B_{\phi} / \mu_{0} \rho r$ and (3) the specific total angular momentum $L(a)=\Omega_{*} r_{\mathrm{A}}^{2}=\Omega r^{2}-r B_{\phi} / \eta$ transported away. Here, $r_{\mathrm{A}}$ is the Alfvén cylindrical radius where mass reaches the Alfvén poloidal velocity. The angular velocity of a magnetic surface $\Omega_{*}$ is roughly equal to the angular velocity $\Omega_{0}$ of the mass at the anchoring cylindrical radius $r_{0}$. If the magnetic field is threading the disc one gets $\Omega_{0} \simeq \sqrt{G M / r_{0}^{3}}$ where $M$ is the stellar mass, whereas $\Omega_{0}=\Omega_{\text {star }}$ if it is anchored into a star with a rotating period $T=2 \pi / \Omega_{\text {star }}$. The following dimensionless parameter

$\lambda=\frac{L}{\Omega_{0} r_{0}^{2}} \simeq \frac{r_{\mathrm{A}}^{2}}{r_{0}^{2}}$

is a measure of the magnetic lever arm braking the rotating object (star or disc). This parameter is an essential feature of MHD jet models, and this is the reason why efforts are made to constrain $\lambda$ through observations.

We are interested here in both accretion-powered jets and stellar winds so we need to be able to describe flows that can be substantially pressure-driven. Thus, we allow for the presence of a heat flux $\boldsymbol{q}=\nabla H-\nabla P / \rho$, where $H$ is the usual enthalpy for a perfect gas. The specific heat input along any given magnetic surface is then $\mathcal{F}(s, a)=\int_{s_{0}}^{s} \boldsymbol{q} \cdot \boldsymbol{e}_{\|} \mathrm{d} s^{\prime}$ (Ferreira \& Casse 2004). This term depends on the curvilinear coordinate $s$ and therefore varies along the flow ( $s_{0}$ is the location where mass has been loaded and $\boldsymbol{B}_{\mathrm{p}}=\boldsymbol{B}_{\mathrm{p}} \boldsymbol{e}_{\|}$). The case of cold stellar winds driven by MHD wave pressure gradients may be treated in a similar way, by adding to the heat input $\mathcal{F}(s, a)$ an extra term (see e.g. Eq. (38) in DeCampli 1981), describing the transfer of energy from the turbulent Alfvén waves to the flow. We will denote $\mathcal{F}^{\prime}(s, a)$ this generalized "pressure" term. Including this additional effect, one gets the generalized Bernoulli invariant

$E(a)+\mathcal{F}^{\prime}(s, a)=\frac{v^{2}}{2}+H+\Phi_{\mathrm{G}}-r \Omega_{0} B_{\phi} / \eta$

where $\Phi_{\mathrm{G}}$ is the stellar gravitational potential and $E(a)$ is the total specific energy provided at the base of the jet (equal to the rhs of Eq. (3) evaluated at $s_{0}$ ). Finally, the shape of each magnetic surface (therefore the whole jet) is given by the GradShafranov equation, but it is of no use for obtaining the jet kinematic properties.

Once far away from the driving source its gravitational attraction can be neglected and the following general expressions apply

$$
\begin{aligned}
r v_{\phi} & =\sqrt{G M R_{0}} \delta_{0} \sin ^{2} \theta_{0} \lambda_{\phi} \\
v_{\mathrm{p}} & =\sqrt{\frac{G M}{R_{0}}} \sqrt{\delta_{0}^{2} \sin ^{2} \theta_{0}\left(2 \lambda_{\mathrm{p}}-1\right)-2+\beta}
\end{aligned}
$$

where $R_{0}$ is the spherical radius $\left(r_{0}=R_{0} \sin \theta_{0}\right), \delta_{0}^{2}=\Omega_{0}^{2} R_{0}^{3} / G M$ is the measure of the rotation of the magnetic surface with respect to gravity, $\beta=2\left(\mathcal{F}^{\prime}+H_{0}-H\right) /\left(G M / R_{0}\right)$ varies along the flow and encompasses all pressure effects (both thermal and turbulent Alfvén waves) and where

$$
\begin{aligned}
& \lambda_{\phi}=(1-g) \frac{r^{2}}{r_{0}^{2}} \\
& \lambda_{\mathrm{p}}=\lambda_{\phi} \frac{1+g}{2} \\
& g=1-\frac{\Omega}{\Omega_{0}}=\frac{m^{2}}{m^{2}-1}\left(1-\frac{r_{\mathrm{A}}^{2}}{r^{2}}\right)
\end{aligned}
$$

are signatures of magnetic effects. Here, $g$ measures the drift between the angular velocity of the matter $\Omega$ and that of the magnetic field $\Omega_{*} \simeq \Omega_{0}$, and $m=v_{\mathrm{p}} / V_{\mathrm{Ap}}$ is the poloidal Alfvénic Mach number. These expressions are valid for any stationary MHD model of an axisymmetric, non-relativistic jet.

The function $g$ is a measure of the conversion of magnetic energy into kinetic energy. It increases from almost zero at the footpoint to a maximum value $g_{\infty}$. Powerful MHD jets are those where $g_{\infty} \simeq 1$ (maximum value allowed), meaning an almost complete transfer of magnetic energy and $\lambda_{\mathrm{p}} \simeq \lambda_{\phi}$. In addition, these jets reach high Alfvénic Mach numbers $m \gg 1$ and large radii $r \gg r_{\mathrm{A}}$ so that eventually $\lambda_{\phi} \simeq r_{\mathrm{A}}^{2} / r_{0}^{2}=\lambda$. The latter equality means that all angular momentum has been transferred to the matter, and is what we define as the "asymptotic regime".

At the distance from the source investigated by current observations ( $\geq 30 \mathrm{AU})$, we are likely to be in this asymptotic regime for jets originating close to the stellar surface (stellar winds and $\mathrm{X}$-winds), while $\beta$ has become constant. In the case of disc winds originating from a large radial extension, the asymptotic regime is achieved further out for the outer streamlines. For instance, a field line anchored at $1 \mathrm{AU}$ has not yet reached this asymptotic regime at $z \sim 50 \mathrm{AU}$ (see below). However, solutions that propagate far away, as demanded by observations, have $g>1 / 2$ already satisfied at the Alfvén surface (see e.g. Ferreira 1997). This is enough to obtain $\lambda_{\mathrm{p}}$ and $\lambda_{\phi}$ converging towards a common value, since $(1+g) / 2 \rightarrow 1$ rapidly. This common value is smaller than the real magnetic lever arm $\lambda$ (until the asymptotic regime is reached), but it can be observationally determined (see below). We will hereafter use $\lambda_{\phi}$ to refer to this value.

\subsection{Accretion-powered disc winds}

In the case of extended disc winds and X-winds $\delta_{0}=1$ and $r_{0}=R_{0}\left(\theta_{0}=\pi / 2\right)$. We thus obtain the following expressions beyond the Alfvén surface, where $\lambda_{\mathrm{p}} \simeq \lambda_{\phi}$ :

$$
\begin{aligned}
r v_{\phi} & =\Omega_{0} r_{0}^{2} \lambda_{\phi} \\
v_{\mathrm{p}} & =\Omega_{0} r_{0} \sqrt{2 \lambda_{\phi}-3+\beta} .
\end{aligned}
$$




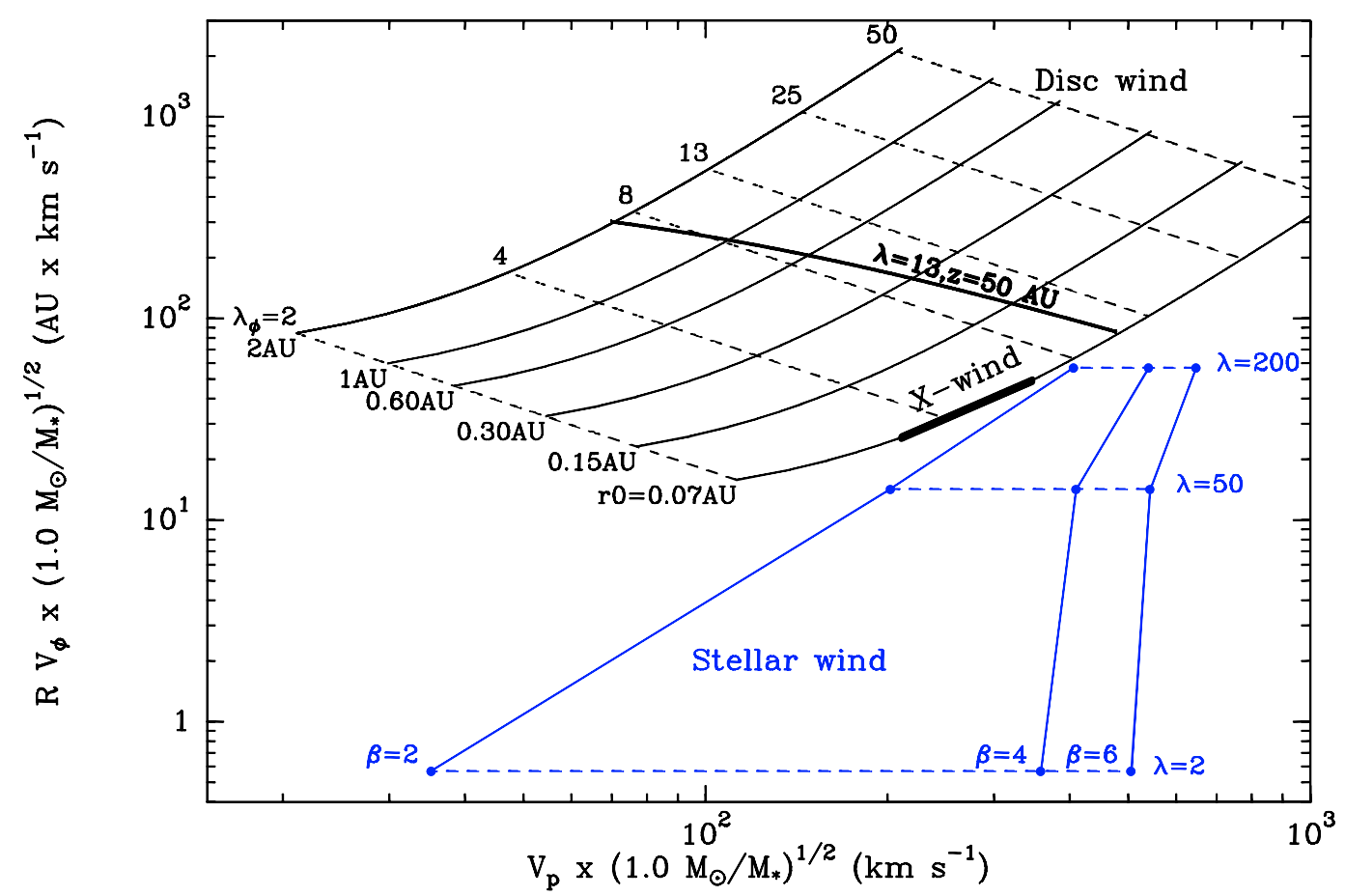

Fig. 2. Relations between specific angular momentum vs. poloidal velocity for all types of stationary MHD jet models, valid once $v_{\mathrm{p}} \gg v_{\phi}$ (e.g. beyond the Alfvén surface). Plotted in solid lines is the relation between $r v_{\phi}$ and $v_{\mathrm{p}}$ for fixed launching radius $r_{0}$ (accretion-powered disc winds; Eq. (11)) or fixed pressure parameter $\beta$ (stellar winds; Eq. (5) with typical T Tauri parameters). Dashed lines indicate curves of constant $\lambda_{\phi}=r v_{\phi} /\left(\Omega_{0} r_{0}^{2}\right)$ in the disc wind case (Eq. (10)), constant $\lambda=r_{\mathrm{A}}^{2} / r_{0}^{2}$ in the stellar wind case (Eq. (4)). A thick segment at $r_{0}=0.07$ AU illustrates the locus of the X-wind (Shang et al. 1998). We also show as a thick curve a cut at $z=50$ AU of a self-similar disc wind solution with $\lambda=13$ settled from 0.07 to $2 \mathrm{AU}$. It can be readily seen that $\lambda_{\phi}$ gives only a lower limit to the true $\lambda$ at $z=50 \mathrm{AU}$.

The influence of pressure (thermal or waves) gradients $\beta$ in the poloidal velocity of jets from Keplerian accretion discs is barely measurable and can be neglected. Indeed, a Keplerian rotation law requires a negligible radial pressure gradient inside the disc. As a consequence, enthalpy at the disc surface always verifies $H_{0} / \Omega_{0}^{2} r_{0}^{2} \sim(h / r)^{2} \ll 1$. Even models of highly warmed up jets, which imply huge sub-Alfvénic temperatures of up to several $10^{5} \mathrm{~K}$, were obtained with $\beta$ of a few percents only (Ferreira \& Casse 2004). In the case of X-winds, the models published so far also have a negligible heat input (see e.g. Najita \& Shu 1994). Thus, we can safely set $\beta=0$ for all accretion-powered disc winds.

We recall that, although thermal effects have no measurable impact on the jet poloidal speeds, they have a tremendous one on mass loading and therefore on the kinematics. An important relation derived from full solutions of the MHD accretion-ejection flow is

$\lambda \simeq 1+\frac{1}{2 \xi}$ where $\dot{M}_{\mathrm{a}} \propto r^{\xi}$

which relates the magnetic lever arm $\lambda$ to the ejected mass through the local ejection efficiency $\xi$ (Ferreira 1997). The physics are quite simple. The same torque will be applied on the underlying accretion disc either with a large mass loss $\xi$ but small lever arm or a small mass loss and large lever arm. What determines exactly the maximum mass loss is the constraint to obtain super-Alfvénic jets, whereas the minimum mass loss is imposed by the disc vertical equilibrium. In vertically isothermal or adiabatic discs ("cold" models), only a tiny mass flux can be lifted from a Keplerian accretion disc, with typically $\xi \sim 0.01$ or less, giving rise to $\lambda \sim 50$ or more (Ferreira 1997; Casse $\&$ Ferreira 2000a). If there is some heat deposition at the resistive disc upper surface layers ("warm" models), more mass can be loaded onto the field lines, up to $\xi \sim 0.1$ or more (Casse $\&$ Ferreira 2000b). These dense solutions have much smaller magnetic lever arms $\lambda \simeq 6-20$.

Combining Eqs. (7) and (8) with $\beta=0$ we get

$$
\begin{aligned}
\frac{r v_{\phi} v_{\mathrm{p}}}{G M} & =\lambda_{\phi} \sqrt{2 \lambda_{\phi}-3} \\
2 r v_{\phi} \Omega_{0} & =v_{\mathrm{p}}^{2}+3 \Omega_{0}^{2} r_{0}^{2} .
\end{aligned}
$$

Equation (10) allows to observationally derive the value of $\lambda_{\phi}$ independently of $r_{0}$ from observed values of $r v_{\phi}$ and $v_{\mathrm{p}}$ (assuming the stellar mass is known). Conversely, as first noted by Anderson et al. (2003), Eq. (11) is independent of $\lambda_{\phi}$ and allows to derive the launching radius $r_{0}$.

It is interesting to note that Anderson et al. (2003) derived Eq. (11) under the assumption that $v_{\phi} \ll v_{\mathrm{p}}$, while we only assumed $\lambda_{\mathrm{p}} \simeq \lambda_{\phi}$ (i.e. $g>1 / 2$ ). Therefore the two assumptions must be equivalent. Since $v_{\phi} \ll v_{\mathrm{p}}$ is indeed observationally verified in T Tauri jets, we conclude that (i) Eq. (11) applies and (ii) the plasma probed by observations has $g>1 / 2$. Note that, at the Alfvén point along any magnetic surface $v_{\phi} / v_{\mathrm{p}} \simeq 1-g_{\mathrm{A}}$ where $g_{\mathrm{A}}$ is the value of the function $g$ at this point. Thus, the plasma is also likely super-Alfvénic. Moreover, $g_{\mathrm{A}} \simeq I_{\mathrm{A}} / I_{\mathrm{SM}}$, with the poloidal current $I=2 \pi r B_{\phi} / \mu_{0}$. Namely, $g$ evaluated at the Alfvén point measures the electrical current remaining inside the MHD jet. Models with $g_{\mathrm{A}} \sim 1$ (most of the MHD power is still available) are possible either for tiny mass fluxes or for warmed up jets (Casse \& Ferreira 2000b). This is a constraint for accretion-powered jet models.

The diagram in Fig. 2 displays the curves defined by Eqs. (10) and (11) in the $\left(v_{\mathrm{p}}, r v_{\phi}\right)$ plane, for $\lambda_{\phi}$ varying from 2 to 50 (dashed curves), and an anchoring radius $r_{0}$ varying 
from 0.07 to $2 \mathrm{AU}$ (solid curves), illustrating the range expected for extended atomic disc winds ${ }^{4}$.

Note that as the plasma accelerates along one particular magnetic surface, its position in the diagram moves to the right along the corresponding $r_{0}=$ const. curve (once $v_{\mathrm{p}} \gg v_{\phi}$ ), and converges asymptotically towards $\lambda_{\phi}=\lambda$. However, an observation is actually a cut across the jet at some altitude $z$, i.e. it samples the whole range of nested magnetic surfaces in the disc wind (if angular resolution is sufficient).

To illustrate the typical locus of an extended disc wind in this diagram, we plot in Fig. 2 a transverse cut at $z=50 \mathrm{AU}$ through a self-similar solution with $\lambda=13$, made of surfaces anchored from $r_{0}=0.07$ to $2 \mathrm{AU}$ (thick solid curve). Because of self-similarity, outer streamlines reach the asymptotic regime at larger distances than inner streamlines. As a consequence, the discrepancy between $\lambda_{\phi}$ and $\lambda$ at a given altitude is larger for outer streamlines. It reaches a factor 2 for $r_{0} \geq 2 \mathrm{AU}$ at $z=$ $50 \mathrm{AU}$ for the solution considered here. This introduces a bias in the determination of $\lambda$, and the locus of a self-similar disc wind deviates slightly from the theoretical $\lambda_{\phi}=\lambda$ curve.

The locus of the X-wind is shown as a thick segment in Fig. 2. This locus is a small fraction of that occupied by extended disc winds. As an X-wind is an accretion-powered jet launched from a tiny interval of radii $r_{0}$ near the inner disc edge, the angular momentum is at the low end of the accessible range. The range in poloidal velocities is also narrower $(\lambda \simeq 3-6$, e.g. Shang et al. 1998). There is another interesting characteristic difference: whatever the shape of the Alfvén surface for the X-wind, the curve drawn by measuring $r v_{\phi}$ and $v_{\mathrm{p}}$ at several transverse radii $r$ across the jet (at a given altitude $z$ ) will always fall on the same $r_{0}=$ const. curve, and will thus have a positive slope in the $\left(v_{\mathrm{p}}, r v_{\phi}\right)$ plane. Note that the same will be true of the ReX-wind component, since it is launched also from near corotation. On the contrary, an extended disc wind is likely to have a negative slope in this plane, as in the self-similar situation with constant $\lambda$ illustrated in our $z=50 \mathrm{AU}$ cut. In order to obtain a positive slope, the extended disc wind would need to have a markedly larger magnetic lever arm $\lambda$ at smaller launching radii. This is very unlikely for it would require an ejection efficiency decreasing towards the central star (one usually expects the contrary).

Therefore, the global amount of angular momentum, and the slope of the $r v_{\phi}$ vs. $v_{\mathrm{p}}$ relation across the jet at a fixed altitude $z$, both offer good discriminants between MHD winds from the corotation (X-wind and ReX-wind) and extended disc winds.

\subsection{Stellar winds}

The typical rotation period of T Tauri stars is 8 days (see Bouvier et al. 1997, and references therein) and corresponds to $\delta_{0} \simeq 0.1$. Moreover, stellar winds are probably only launched at large latitudes $\left(\sin \theta_{0}<1 / 2\right)$, higher anyway than those where accreting material is flowing in. Thus, Eq. (5) shows that magnetocentrifugal acceleration would be efficient only if the stellar field lines are wide open with a very large magnetic lever arm parameter, namely $\lambda>1 / \delta_{0}^{2} \sin ^{2} \theta_{0} \sim 200$. Although it has been recently proposed that such winds could solve the angular momentum problem in T Tauri stars (Matt \& Pudritz 2005), there is no MHD calculation yet showing the feasibility of such a flow for

4 The $\lambda_{\phi}$ values span a range corresponding to the self-similar "warm" disc wind solutions investigated by Casse \& Ferreira (2000b). Warm solutions have been found numerically for $\lambda \geq 6$ but, since the condition of a positive Bernoulli invariant requires only $\lambda>3 / 2$, and $\lambda_{\phi} \leq \lambda$, we plot curves from $\lambda_{\phi}=2$ for completeness. typical magnetic field strengths and jet mass-loss rates in T Tauri stars.

Instead, all self-similar models of MHD stellar winds computed so far have an Alfvén surface located not very far from the stellar surface. Such winds have almost straight field lines in the sub-Alfvénic zone so that $\lambda=r_{\mathrm{A}}^{2} / r_{0}^{2} \simeq R_{\mathrm{A}}^{2} / R_{0}^{2}$, where $R_{0}$ is the stellar radius. For instance, model I of Sauty \& Tsinganos (1994) displays $R_{\mathrm{A}}=1.55 R_{0}(\lambda \simeq 2.4)$, whereas Trussoni et al. (1997) obtained a solution with $R_{\mathrm{A}}=7.1 R_{0}(\lambda \simeq 50)$. As a consequence, published MHD stellar wind models are essentially pressure-driven winds where the ejected plasma gets most of its energy from $\beta$. Note that magnetic fields do play a role in collimating the outflow, but this collimation takes place mainly beyond the Alfvén surface.

Figure 2 illustrates the locus of MHD stellar winds from T Tauri stars, using the conservative value $\delta_{0} \sin ^{2} \theta_{0}=0.04$ and a stellar radius $R_{0}=3 R_{\odot}$, for $\beta$ values varying from 2 to 6 (solid lines) and $\lambda$ from 2 to 200 (dashed lines) for the sake of completeness. Stellar winds occupy only a narrow region of the $\left(v_{\mathrm{p}}, r v_{\phi}\right)$ plane of MHD models (note this is a log-log plot). They are well separated from MHD disc winds, with only those stellar winds with very large $\lambda>100$ approaching the $\mathrm{X}$-wind region.

For a given $\lambda$ value, stellar winds are characterized by straight horizontal lines corresponding to the observable specific angular momentum:

$r v_{\phi}=\delta_{0} \sin ^{2} \theta_{0} \lambda \sqrt{G M R_{0}}$

Figure 2 shows that, for published stellar wind models where $\lambda=$ $2-50$, no detectable signature of rotation is expected from slowly rotating young stars. For the same range of $\lambda$, and contrary to accretion-powered disc winds, the asymptotic poloidal velocity of stellar winds almost entirely depends on the asymptotic value of $\beta(>2)$ alone (cf. Eq. (5)), namely

$v_{\mathrm{p}} \simeq 250 \sqrt{\beta-2}\left(\frac{M}{M_{\odot}}\right)^{1 / 2}\left(\frac{R_{0}}{3 R_{\odot}}\right)^{-1 / 2} \mathrm{~km} \mathrm{~s}^{-1}$.

As an example, model I of Sauty \& Tsinganos (1994) used $\beta=6.64$ giving a terminal speed of $543 \mathrm{~km} \mathrm{~s}^{-1}$. Figure 2 clearly shows that only a much larger value of $\lambda(\geq 200)$ introduces a deviation from the simple expression given by Eq. (13).

\section{Comparison with T Tauri microjets observations}

We now compare our kinematic predictions for stationary selfconfined jet models with recent high-angular observations of the inner regions of $\mathrm{T}$ Tauri microjets, using the diagnostic diagram proposed in Fig. 2. The resulting implications for disc winds and stellar winds are discussed below.

\subsection{Rotation signatures}

Detections of transverse velocity shifts suggestive of rotation signatures have been recently derived from HST/STIS spectra at distances $\simeq 50 \mathrm{AU}$ from the central source in $4 \mathrm{~T}$ Tauri microjets: DG Tau, Th 28, LkH $\alpha$ 321, and RW Aur (Bacciotti et al. 2002; Coffey et al. 2004; Woitas et al. 2005). Tentative rotation signatures were previously reported by Davis et al. (2000) in the younger $\mathrm{HH} 212$ jet at $2300 \mathrm{AU}$ from the central infrared source (knot SK1), using the $\mathrm{H}_{2} v=1-0 \mathrm{~S}(1)$ line.

The rotation interpretation appears particularly convincing in the case of the intermediate velocity component (IVC) of the DG Tau jet, as the rotation sense matches that of the disc 


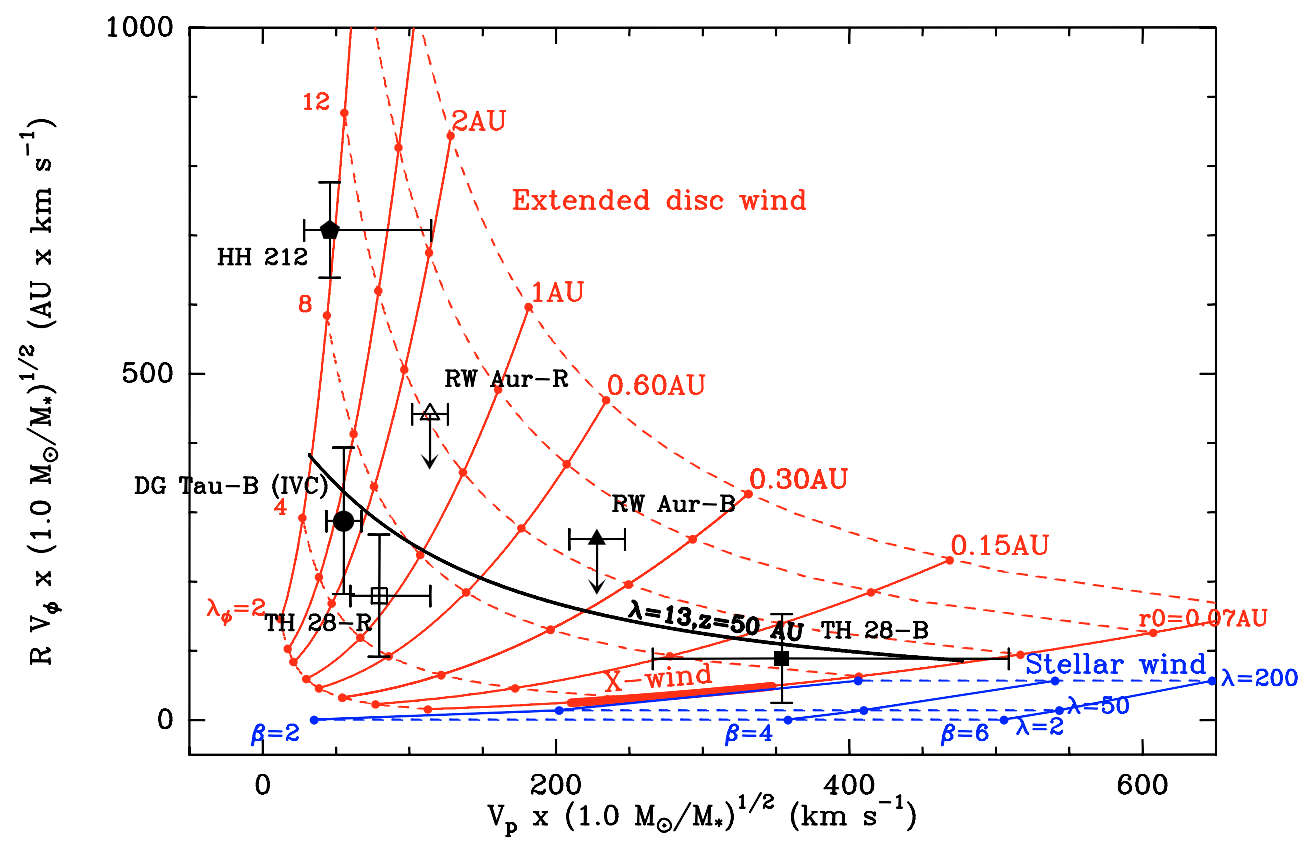

Fig. 3. Comparison of predicted specific angular momentum vs. poloidal velocities with observations of T Tauri microjets. Full and dashed curves show expected theoretical relations for MHD disc and stellar winds (same as Fig. 2, on a linear scale). Plotted in symbols are jet kinematics measured at distance $z \simeq 50 \mathrm{AU}$ in the DG Tau, RW Aur, and Th 28 jets. The infrared HH 212 jet is also shown for comparison. See text for more details on how the data points and their associated error bars are computed.

(Testi et al. 2002) and transverse shift variations across and along the jet are in excellent agreement with detailed predictions for a full MHD solution of an extended disc wind (Pesenti et al. 2004).

Care must however be exercised as currently detected velocity shifts are close to the detection limit and are thus affected by large uncertainties. Additional effects, such as intrinsic asymmetric velocity structure in the flow and/or contamination by entrained material, cannot be fully excluded. Detected velocity shifts may therefore correspond only to upper limits to the true jet azimuthal velocities. For example, tentative rotation signatures reported by Woitas et al. (2005) in the bipolar RW Aur jet appear to be in opposite sense to the disc rotation (Cabrit et al. 2006), and are thus likely only upper limits.

In Fig. 3, we plot the derived specific angular momentum versus poloidal velocities for all of the above jets (except $\mathrm{LkH} \alpha 321$, for which inclination is unknown), with distinct symbols for redshifted and blueshifted lobes. In this graph, we consider only significant measurements obtained towards outer jet streamlines at transverse distances $d_{\perp} \geq 20 \mathrm{AU}$ from the jet axis, as detailed modelling shows that more axial jet regions are heavily affected by projection and beam dilution effects, leading to severe underestimation of their azimuthal velocity (Pesenti et al. 2004). As a consequence, current observations are only probing the launching radius $r_{\mathrm{e}}$ of the outer streamlines of the optical jet. For the DG Tau microjet, we average velocity shifts and radial velocities reported by Bacciotti et al. (2002) at $d_{\perp}=30 \mathrm{AU}$ for distances along the jet between 40 and 60 AU. For the RW Aur microjet, we adopt the values of $v_{\phi}$ and $v_{\mathrm{p}}$ published by Woitas et al. (2005, their Table 1). However, given the discrepancy between jet and disk rotation sense, we take these values as upper limits. For the Th 28 redshifted flow, we average velocity shifts and radial velocities observed by Coffey et al. (2004) at $d_{\perp}=34 \mathrm{AU}$ in the [O I] $6300 \AA$ and [S II] 6716, $6731 \AA$ lines. For the Th 28 blueshifted flow, we take the measurements at $d_{\perp}=25 \mathrm{AU}$ in the $[\mathrm{N} \mathrm{II}] 6583 \AA$ line.
Table 1 summarizes the adopted values of stellar mass, jet inclination $i$, and $v_{\mathrm{p}}$ and $v_{\phi}$ at transverse distance $d_{\perp}$ together with their error bars. To place observed data points in Fig. 3, we have taken into account a $10 \%$ (DG Tau, RW Aur) to $20 \%$ (Th 28, HH 212) uncertainty on the value of the central stellar mass.

\subsection{Constraints on wind launching radii and magnetic lever arms}

Several general conclusions may be drawn from the comparison of data points with model predictions in Fig. 3. (i) Dynamically cold disc winds (with magnetic lever arms $\lambda \geq 50$ ) are excluded (in agreement with the conclusions of Garcia et al. 2001; Pesenti et al. 2004), since they would predict rotation rates largely in excess of what is observed; (ii) X-winds and stellar winds predict 10-100 times smaller angular momentum than suggested by current tentative rotation signatures in TTS jets; (iii) if these signatures indeed trace pure rotation in the jet material, then extended disc winds with $r_{\mathrm{e}}$ of $\simeq 0.2$ to $3 \mathrm{AU}$ and moderate magnetic lever arm parameters $\lambda_{\phi} \simeq 4-18$ (corresponding to "warm" solutions) are needed. In particular, it is interesting to note that most of the current measurements, including the upper limits in the RW Aur jet, appear roughly compatible with the extended MHD disc wind model with $\lambda=13$ that fits (Pesenti et al. 2004) the DG Tau jet dataset (thick curve in the figure).

The values of $\lambda_{\phi}$ and $r_{\mathrm{e}}$ inferred from comparison with disc wind predictions using Eqs. (10) and (11) are listed in Table 1. Although derived only from the most reliable subset among available data (see previous section), they remain within the error bars of previously published estimates (Bacciotti et al. 2002; Anderson et al. 2003; Coffey et al. 2004; Woitas et al. 2005).

We recall that if detected velocity shifts include other effects than rotation, they give only upper limits to the true jet azimuthal velocities. The derived launching radii and magnetic lever arms are then also upper limits to the true disc wind 
Table 1. Kinematics of $\mathrm{T}$ Tauri jets with rotation estimates and derived disc wind parameters.

\begin{tabular}{lccccccccc}
\hline \hline Jet & $\begin{array}{c}M_{*} \\
\left(M_{\odot}\right)\end{array}$ & $i$ & $\begin{array}{c}v_{\mathrm{p}}^{a, b} \\
\left(\mathrm{~km} \mathrm{~s}^{-1}\right)\end{array}$ & $\begin{array}{c}v_{\phi}^{a} \\
\left(\mathrm{~km} \mathrm{~s}^{-1}\right)\end{array}$ & $\begin{array}{c}d_{\perp} \\
(\mathrm{AU})\end{array}$ & Refs. $^{c}$ & $\lambda_{\phi}$ & $\begin{array}{c}r_{\mathrm{e}} \\
(\mathrm{AU})\end{array}$ & $\left(\dot{M}_{\mathrm{j}} / \dot{M}_{\mathrm{a}}\right)_{\max }^{d}$ \\
\hline DG Tau-Blue IVC & 0.67 & $45^{\circ} \pm 3^{\circ}$ & $45 \pm 9$ & $7.8 \pm 3.0$ & 30. & 1 & $4-8$ & $1.5-4.5$ & 0.3 \\
RW Aur-Blue & 1.3 & $46^{\circ} \pm 3^{\circ}$ & $260 \pm 17$ & $<15$. & 20. & 2,3 & $<15$ & $<0.5$ & - \\
RW Aur-Red & 1.3 & $46^{\circ} \pm 3^{\circ}$ & $130 \pm 12$ & $<17$. & 30. & 2,3 & $<13$ & $<1.7$ & - \\
Th 28-Blue & $1:$ & $80^{\circ} \pm 3^{\circ}$ & $354_{-80}^{+150}$ & $3.5 \pm 2.5$ & 25. & 4 & $4-16$ & $0.03-0.2$ & 0.06 \\
Th 28-Red & $1:$ & $80^{\circ} \pm 3^{\circ}$ & $80_{-18}^{+33}$ & $5.3 \pm 2.5$ & 34. & 4 & $3.5-9$ & $0.5-2$ & 0.3 \\
HH 212 (SK1) & $1:$ & $85^{\circ} \pm 3^{\circ}$ & $46_{-17}^{+68}$ & $1.5 \pm 0.25$ & 470. & 5 & $7-17$ & $2-12$ & 0.2 \\
\hline
\end{tabular}

\begin{abstract}
${ }^{a}$ Velocities $v_{\mathrm{p}}$ and $v_{\phi}$ were computed from the observed radial velocities and velocity shifts at transverse distance $\pm d_{\perp}$ using the following expressions: $v_{\mathrm{p}}=v_{\mathrm{rad}} / \cos (i)$ and $v_{\phi}=v_{\text {shift }} / 2 \sin (i)$, where $i$ is the inclination of the jet axis to the line of sight. ${ }^{b}$ Our quoted uncertainties on $v_{\mathrm{p}}$ take into account a typical $3^{\circ}$ uncertainty on the inclination angle. This latter source of error is particularly important in the case of Th 28 and HH 212 where a high inclination of $80^{\circ}-85^{\circ}$ is inferred. ${ }^{c}$ References: [1] Bacciotti et al. (2002); [2] Woitas et al. (2005); [3] this paper; [4] Coffey et al. (2004); [5] Davis et al. (2000). ${ }^{d}$ One-sided mass ejection to accretion ratio.
\end{abstract}

parameters. Therefore, $\mathrm{X}$-winds and stellar winds cannot be definitely ruled out on the basis of current rotation measurements which sample only the outer jet. Determination of the full transverse jet rotation profile would be a crucial test, as demonstrated in Sect. 3. This will require better angular resolution $(<5 \mathrm{AU})$ and tracers of higher critical density (Pesenti et al. 2004).

\subsection{Poloidal velocities}

Independently of rotation measurements, the wide range of poloidal velocities observed across $\mathrm{T}$ Tauri jets and sometimes within the same jet $\left(50-400 \mathrm{~km} \mathrm{~s}^{-1}\right)$ also raises interesting issues for the jet launching mechanism.

$\mathrm{X}$-wind models launched from the disc corotation radius $r_{\mathrm{co}}$ produce, by construction, only a relatively narrow range of poloidal speeds. For the Alfvén surface calculated in e.g. Shang et al. (1998), where $\lambda \simeq 3-6$, one obtains

$v_{\mathrm{p}} \times\left(1 M_{\odot} / M_{*}\right)^{0.5} \simeq 200-340\left(0.07 \mathrm{AU} / r_{\mathrm{co}}\right)^{0.5} \mathrm{~km} \mathrm{~s}^{-1}$.

Although this range agrees with a good fraction of known T Tauri jets (Hartigan et al. 1995), it fails to explain the intermediate velocity component (IVC) at $v_{\mathrm{p}}<100 \mathrm{~km} \mathrm{~s}^{-1}$ which often dominates the emission towards optical jet edges, as e.g. in the DG Tau, Th 28-red, and RW Aur-red jets (Lavalley-Fouquet et al. 2000; Bacciotti et al. 2000; Coffey et al. 2004; Woitas et al. 2005). Reproducing these observations with an X-wind would require a substantial change in the Alfvén surface geometry, with $\lambda \leq 2$ for equatorial streamlines. Alternatively, the IVC could be attributed to entrainment of ambient gas. However, given the wide opening angle of the $\mathrm{X}$-wind, it is unclear whether this process could be efficient within 20-30 AU of the jet axis, where the IVC is observed. In either case, a substantial modification of the current published solutions would be needed.

In contrast with $\mathrm{X}$-winds, extended disc winds with $\lambda \simeq 10$ easily reproduce the whole range of poloidal speeds observed in T Tauri jets. As can be seen in Fig. 3, material ejected from launching radii beyond $0.5 \mathrm{AU}$ naturally explains the intermediate velocity components at $v_{\mathrm{p}}<100 \mathrm{~km} \mathrm{~s}^{-1}$ seen towards jet edges, while material ejected close to the disc corotation radius $\sim 0.07$ AU reaches high speeds of up to $400-500 \mathrm{~km} \mathrm{~s}^{-1}$ sufficient to explain the fastest jets observed in TTS. Hence, within a given jet lobe, a disc wind will naturally provide a range in poloidal jet velocities because of the range of Keplerian speeds in the launching zone. Assuming $\lambda$ constant throughout the extended disc wind, a factor 10 in launching radii will produce a drop of a factor 3 in observed velocities, from jet axis to jet edge. This is roughly the magnitude of the decline in poloidal speed from axis to edge across the DG Tau and Th 28-red jets (Lavalley-Fouquet et al. 2000; Bacciotti et al. 2000; Coffey et al. 2004).

Finally, in the case of stellar winds, Fig. 3 shows that the range of jet poloidal speeds can easily be reproduced with $\beta \simeq 2-4$. To get an insight of the energy reservoir required per particle, let us first assume that $\beta$ is mostly provided by an initial enthalpy. In this case, the initial jet temperature must be

$T_{0}=1.510^{6}\left(\frac{\beta}{2}\right)\left(\frac{M}{M_{\odot}}\right)\left(\frac{R_{0}}{3 R_{\odot}}\right)^{-1} \mathrm{~K}$.

Since $\beta>2$ (Eq. (13)), this implies that ejected material must have coronal temperatures. Such a high temperature could be provided either by a magnetic coronal activity or by the accretion shock onto the stellar surface. Indeed, the post-shock temperature of an adiabatic hydrodynamic shock is

$T=\frac{8}{9} \frac{m_{\mathrm{p}}}{k_{\mathrm{B}}} v_{\mathrm{s}}^{2} \simeq 9.3 \times 10^{6}\left(\frac{v_{\mathrm{s}}}{300 \mathrm{~km} \mathrm{~s}^{-1}}\right)^{2} \mathrm{~K}$

for a fully ionized gas, where $v_{\mathrm{s}}$ is the velocity of freely falling material onto the TTS. If $\beta$ is constructed by such a shock, then this could explain the accretion-ejection correlation. Note however that the above equation is an optimistic estimate, since infalling material would certainly be cushioned by stellar magnetic fields, thus lowering the compression and the resulting temperature. Moreover, these shocks are radiating and matter that becomes ultimately loaded onto open field lines must not cool too rapidly while diffusing towards the higher latitude open field lines. TTS with prominent coronal winds do show reduced optical continuum emission from the accretion shock (Beristain et al. 2001), in line with this kind of scenario. But the major problem with such a scenario is the unavoidable radiative losses from the densest parts: if $\beta$ is in the form of initial enthalpy, then mass loss rates above $10^{-9} M_{\odot} /$ yr would radiate more X-rays than observed (DeCampli 1981).

To circumvent this problem, one has to assume that the driving pressure effects modeled by $\beta$ are mostly provided along the flow and not at the base. For instance, a sophisticated stellar wind model of Sauty \& Tsinganos (1994) has an initial temperature $T_{0} \sim 10^{4} \mathrm{~K}$ which then raises extremely sharply to $10^{6} \mathrm{~K}$ and levels off to several $10^{6}-10^{7} \mathrm{~K}$ thanks to the heating term $\mathcal{F}$ (see their Fig. 9). The computation of the X-ray emission of such a 
wind remains to be done but it is expected to be much smaller in this case. The same conclusion holds for cold wave-driven winds where $\beta \simeq 2-4$ is provided by the transfer of turbulent wave energy.

\subsection{Mass-loss rates}

As noted in Sect. 3.2, the magnetic lever arm in disc winds is intrinsically related to the local ejection efficiency $\xi$ by $\lambda \simeq 1+$ $1 / 2 \xi$ (Eq. (9)). Furthermore, observations of $v_{\phi}$ and $v_{\mathrm{p}}$ provide a lower limit $\left(\lambda_{\phi}\right)$ on the magnetic lever arm $\lambda$, therefore an upper limit on $\xi$. This information, combined with the knowledge of the maximum outer anchoring radius $r_{\mathrm{e}}$ (Table 1), gives an upper limit on the (one-sided) mass ejection to accretion ratio that can be sustained by an extended disc wind in each object:

$\frac{\dot{M}_{\mathrm{j}}}{\dot{M}_{\mathrm{a}}} \leq \frac{1}{4\left(\lambda_{\phi}-1\right)} \ln \frac{r_{\mathrm{e}}}{r_{\mathrm{i}}}$.

The last column in Table 1 lists the maximum ratios obtained for each of the observed TTS jets, assuming a constant $\lambda$ throughout the disc and $r_{\mathrm{i}} \sim 0.1 \mathrm{AU}$ (corotation radius for a typical $\mathrm{T}$ Tauri star).

In principle, these values could be checked for consistency against the observationally determined ejection/accretion ratio in the same objects. Conversely, a lower limit to the observed mass flux ratio could in principle be used to derive an upper limit on $\lambda$ (see Woitas et al. 2005). However, such a comparison made on a star by star basis is not as constraining as one would hope, as both jet mass-loss rates and accretion rates currently suffer from large uncertainties: Four different methods exist in the literature to derive jet mass-loss rates from observed line fluxes and jet sizes, and they give results that typically differ by a factor 5-10 (see Hartigan et al. 1995, Cabrit 2002). As for accretion rates, their derivation from optical veiling involves a series of complex steps, with discrepancies of up to a factor 10 between different authors (Hartigan et al. 1995; Gullbring et al. 1998). Furthermore, veiling varies on much shorter timescales (days) than those probed by current jet observations $(\sim 1 \mathrm{yr}$ at $z=50 \mathrm{AU})$, introducing an intrinsic scatter in the ejection/accretion ratio for TTS jets. Therefore, a statistical comparison is probably more meaningful at this stage. On average, the observed ratio of (one-sided) jet mass-flux to disc accretion rate in TTS lies in the range $0.01-0.1$, depending on the adopted accretion rates, with a scatter of a factor 3-10 (Cabrit 2002). This range is in excellent agreement with the values listed in Table 1, indicating that indeed magnetic disc wind models accounting for $\mathrm{T}$ Tauri jet kinematics are also able to sustain the observed jet mass-loss rates in these sources.

This is not so clear for stellar winds: the requirement that all the ejected mass gains a large specific energy through pressure gradients $(\beta)$ puts a severe energetic constraint. Indeed, the total power transferred to the two jets via either thermal or wave pressure gradients is simply

$L_{\beta} \simeq \bar{\beta} \frac{G M \dot{M}_{\mathrm{j}}}{R_{*}} \simeq \bar{\beta}\left(\frac{\dot{M}_{\mathrm{j}}}{\dot{M}_{\mathrm{a}}}\right) L_{\mathrm{acc}}$

where $\bar{\beta}$ is the average value of $\beta$ through the jet and $L_{\text {acc }}=$ $G M \dot{M}_{\mathrm{a}} / R_{*}$ the accretion luminosity onto the star. Taking $\bar{\beta}=3$ and a one-sided ejection to accretion mass ratio ranging from $1 \%$ to $10 \%$ gives a total power that must be as high as $3 \%$ to $30 \%$ of the accretion luminosity. But this transferred power is itself a fraction of the total power that must be available to the ejected material. Since this power is presumably stored in some accretion-related turbulence, we can write $L_{\beta}=\eta L_{\text {turb }}$ where $\eta$ is the efficiency of energy conversion. For instance DeCampli (1981) obtained an efficiency of only roughly $20 \%$ with a prescribed radial field and assuming undamped waves. This is a conservative value considering that the ejected plasma will also loose energy though radiation. Thus, the total net power $L_{\text {turb }}$ that must be available for stellar winds must be as high as $15 \%$ to $150 \%$ of the accretion luminosity! Clearly this is very uncomfortable.

Note that this poses an energetic problem only if one insists on explaining all the jet mass loss with stellar winds. This probably implies that stellar winds only carry a small fraction of the observed jet mass flux in T Tauri stars.

\subsection{The origin of jet asymmetries}

An intriguing property of T Tauri jets is the frequent occurence (in more than half the cases) of a strong asymmetry of up to a factor 2 in poloidal speed between the two jet lobes (Hirth et al. 1994), as may be seen in Table 1 for RW Aur and Th 28. One might think it difficult to obtain such asymmetries within MHD jet models. We argue below that, in fact, all models may in principle account for them, asymmetries representing only another set of constraints.

In the frame of stellar winds, such a velocity asymmetry could be obtained with a difference of a factor 2 in the pressure parameter $\beta$ between the two stellar poles (see Fig. 3). Further testing of this hypothesis requires to elucidate the origin of the pressure gradients, which remains the major unsolved issue in stellar wind models for TTS.

In the frame of MHD disc winds, jet asymmetries require an asymmetry in magnetic lever arms, or launch radii, or both, between either sides of the disc. Such a situation may occur naturally in an asymmetric ambient medium. Indeed, an accretionejection structure can be seen as a rotating wheel (the disc) with two independent electric circuits (the jets). The current that flows in each circuit depends on the power developed by the wheel (which is fixed) but also on the electric resistance which may be different on each side (Ferreira \& Pelletier 1995; Ferreira 2002). Thus, whenever the ambient medium leads to an asymmetric interaction (i.e. dissipation) with the bipolar jets, then one should expect a different total current flowing at each disc surface, namely different current densities (namely the toroidal magnetic field, affecting $\lambda$ ) and/or different radial extensions (affecting $r_{\mathrm{e}}$ ).

Another but equivalent way to look at it is that one necessary condition for jet production is the presence of a large scale vertical magnetic field close to equipartition in the disc (Ferreira \& Pelletier 1995). But this is not a sufficient condition, as mass must be able to escape from the turbulent accretion disc. Now, the conditions for this escape can differ from one disc surface to the other, since they are strongly affected by heat deposition at the surface layers (Casse \& Ferreira 2000b). For instance, if the ambient radiation field is stronger in one side, then heating and ionization can be more important, leading to enhanced mass flux (smaller $\lambda$ ) and/or a larger jet launching domain. Note also that heating of the disc surface layers could be provided by dissipation of upstream waves, triggered by the interaction between the jet and its environment. Any asymmetry of this medium may therefore also lead to an asymmetric ejection. 


\section{Summary and implications for the ejection mechanisms in young stars}

We have presented theoretical arguments demonstrating that unsteady MHD ejections from the magnetosphere/disc interface, although probably present in $\mathrm{T}$ Tauri systems, may not contribute a dominant fraction of the mass-flux in $\mathrm{T}$ Tauri optical jets.

To further quantify constraints on the driving mechanism for TTS jets, we have extended upon the work of Anderson et al. (2003), and presented predictions for toroidal and poloidal velocities in all types of stationary self-collimated MHD jet models, namely accretion-driven disc winds and pressure-driven stellar winds, valid as soon as $v_{\mathrm{p}} \gg v_{\phi}$.

We have found that the location in the $\left(v_{\mathrm{p}}, r v_{\phi}\right)$ plane is a general and powerful diagnostic to discriminate among MHD models and derive relevant parameters. Comparison of model predictions with recent observations of jet kinematics within $\sim 200 \mathrm{AU}$ of their source yield several results:

- Extended "cold" disc wind models are excluded in TTS (in line with the conclusion of Garcia et al. 2001) as their large magnetic lever arm $(\lambda \geq 50)$ predicts excessive jet rotation on observed scales.

- Published stellar wind models for TTS predict a very small specific angular momentum $\simeq 5(\lambda / 50)$ AU $\mathrm{km} \mathrm{s}^{-1}$, roughly 60-100 times smaller than current observational estimates/upper limits in TTS jets. Moreover, the jet poloidal speeds can only be reproduced with a large specific energy deposition, on the order of $1-2 \times G M / R_{*}$. Such a large input of additional energy raises the question of the origin of this energy reservoir if stellar winds were to carry all the observed jet mass loss. On the other hand, there is mounting evidence for the presence of a hot inner wind close to the stellar surface in TTS (Edwards et al. 2003; Dupree et al. 2005). We propose that MHD stellar winds do contribute as an axial flow inside $\mathrm{T}$ Tauri jets but carry only a small fraction of the total mass loss.

- Published wide-angle disc winds from the corotation ("X-winds") predict a moderate specific angular momentum $\simeq 50 \mathrm{AU} \mathrm{km} \mathrm{s}{ }^{-1}$, roughly 10 times smaller than current observational estimates/upper limits in TTS jets. Moreover, the range in poloidal speeds is narrower than observed (50$400 \mathrm{~km} \mathrm{~s}^{-1}$ ), and the frequent steep decline in poloidal speed towards jet edges is not explained. Solving these problems would require a strong modification in the Alfvén surface and/or in the collimation of outer streamlines (to allow entrainment of slow ambient gas within 20-30 AU of the jet axis).

- Extended "warm" disc winds with moderate lever arms $\lambda \simeq 13$ (Casse $\&$ Ferreira 2000b) predict a range in angular momentum and poloidal speeds readily compatible with current observations of TTS jets, even when asymmetric, provided they are launched from an inner radius $r_{\mathrm{i}} \simeq 0.1 \mathrm{AU}$ (corotation) out to an external radius $r_{\mathrm{e}}$ ranging from 0.2 to several AU. Such extended disc winds also reproduce the observed ejection to accretion ratios, as well as the jet collimation and line profile shapes (Pesenti et al. 2004). Accurate determination of the full transverse rotation profile in the jet is however critically needed, as current estimates of rotation speeds and launching radii are otherwise only upper limits.

We therefore favor the extended disc wind scenario as the currently simplest explanation for the main component in jets from T Tauri stars. Moreover, (1) it produces self-collimated jets able to (2) provide a pressure confining any plasmoid ejected at the magnetopause (either Reconnection X-winds or CME-like ejecta) and (3) is hollow, allowing the propagation of an inner pressure-driven stellar wind. We therefore expect all these components to be simultaneously present but most of the mass being carried by the disc wind. We note that although a stellar wind may be unable to carry a large fraction of the ejected mass, it allows to carry the returning electric current needed to confine the outer disc wind ${ }^{5}$. Within this framework, variability phenomena on time scales smaller than or comparable to the stellar rotational period should be interpreted as signatures of the star-disc interaction. In particular, if the stellar magnetic moment is parallel to the disc field, efficient braking of the contracting star is possible through a ReX-wind (Ferreira et al. 2000).

The hypothesis of extended MHD disc winds in T Tauri stars has several important implications on accretion disc physics. To obtain solutions with moderate lever arms $(\lambda \sim 10)$, a necessary condition is heat input at the upper disc surface layers. Such an input in the resistive MHD disc region allows more mass to be loaded onto the field lines ( $\xi \sim 0.1$, Casse \& Ferreira 2000b). The origin of this heat deposition remains an open question. It cannot be due solely to illumination by stellar UV and X-ray radiation (Garcia et al., to be submitted). Alternatively, the turbulent processes responsible for the required magnetic diffusivity inside the disc might also lead to a turbulent vertical heat flux leading to dissipation at the disc surface layers. It is interesting to note that in current MHD simulations of the magneto-rotational instability a magnetically active "corona" is quickly established (Stone et al. 1996; Miller \& Stone 2000). Although no 3D simulation has been done with open magnetic field lines, this result is rather promising. Indeed, it might be an intrinsic property of the MHD turbulence in accretion discs, regardless of the launching of jets (see arguments developed by Kwan 1997, and Glassgold et al. 2004).

The prime condition for the existence of accretion powered disc winds (extended or X-winds) is the presence of a vertical large scale magnetic field close to equipartition (Ferreira \& Pelletier 1995), namely

$$
B_{z} \simeq 0.2\left(\frac{M}{M_{\odot}}\right)^{1 / 4}\left(\frac{\dot{M}_{\mathrm{a}}}{10^{-7} M_{\odot} / \mathrm{yr}}\right)^{1 / 2}\left(\frac{r_{0}}{1 \mathrm{AU}}\right)^{-5 / 4+\xi / 2} \mathrm{G}
$$

threading the disc on some extension (up to $r_{\mathrm{e}}$ ). Thus, probing the external launching radius $r_{\mathrm{e}}$ of jets from YSOs is an indirect way to constrain the disc magnetic field. The origin of this field is still a matter of debate. It could be either advected from the parent molecular cloud or locally generated by a dynamo (or both). The amount of the magnetic flux threading the disc is thus an issue in star formation, but it is likely to vary from one object to another.

Ferreira et al. (2000) showed that contracting protostars initially rotating at break-up speeds can be spun down via ReX-winds, on the duration of the embedded phase, to low rotational speeds characteristic of TTS. But the natural outcome of this model is the decline in time of the disc magnetic flux. More precisely, the disc field reconnects with closed stellar field lines and, eventually, ends up as open magnetic lines anchored on the star. Thus, according to this picture, the relative importance of extended disc winds should decrease in time $\left(r_{\mathrm{e}} \rightarrow r_{\mathrm{i}}\right)$. Ultimately, within this framework, some slowly rotating TTS

\footnotetext{
5 The electric circuit envisioned is flowing on the axis towards the star, enters the disc at its inner edge (the magnetopause) and leaves it at the disc surface (inside the jet).
} 
should only drive stellar winds (along with some unsteady magnetospheric events). In this respect, it is particularly interesting to note that some TTS have indeed no spectroscopic evidence for jets in the form of high-velocity components in forbidden lines (Hartigan et al. 1995). They exhibit only a low-velocity component in [O I] not clearly correlated with accretion, possibly tracing a photo-evaporating layer at the disc surface. Such an evolutionary effect needs testing on a broad sample of young stellar objects. Our proposed diagram will then be a prime diagnostic tool to use.

Acknowledgements. S.C.'s visits to LAOG were partly funded by the Programme National de Physique Stellaire. The authors acknowledge support through the Marie Curie Research Training Network JETSET (Jet Simulations, Experiments and Theory) under contract MRTN-CT-2004-005592.

\section{References}

Aly, J. J. 1991, ApJ, 375, L61

Anderson, J. M., Li, Z.-Y., Krasnopolsky, R., \& Blandford, R. D. 2003, ApJ, 590, L107

Bacciotti, F., \& Eislöffel, J. 1999, A\&A, 342, 717

Bacciotti, F., Mundt, R., Ray, T. P., et al. 2000, ApJ, 537, L49

Bacciotti, F., Ray, T. P., Mundt, R., Eislöffel, J., \& Solf, J. 2002, ApJ, 576, 222

Beristain, G., Edwards, S., \& Kwan, J. 2001, ApJ, 551, 1037

Blandford, R. D., \& Payne, D. G. 1982, MNRAS, 199, 883

Bouvier, J., Forestini, M., \& Allain, S. 1997, A\&A, 326, 1023

Burrows, C. J., Stapelfeldt, K. R., Watson, A. M., et al. 1996, ApJ, 473, 437

Cabrit, S. 2002, in Star Formation and the Physics of Young Stars, ed. J. Bouvier, \& J.-P. Zahn, EAS Publications Series, 3, 147

Cabrit, S., Edwards, S., Strom, S. E., \& Strom, K. M. 1990, ApJ, 354, 687

Cabrit, S., Ferreira, J., \& Raga, A. C. 1999, A\&A, 343, L61

Cabrit, S., Pety, J., Pesenti, N., \& Dougados, C. 2006, submitted

Casse, F., \& Ferreira, J. 2000a, A\&A, 353, 1115

Casse, F., \& Ferreira, J. 2000b, A\&A, 361, 1178

Coffey, D., Bacciotti, F., Woitas, J., Ray, T. P., \& Eislöffel, J. 2004, ApJ, 604, 758

Davis, C. J., Berndsen, A., Smith, M. D., Chrysostomou, A., \& Hobson, J. 2000, MNRAS, 314, 241

DeCampli, W. M. 1981, ApJ, 244, 124

Dougados, C., Cabrit, S., Lavalley, C., \& Ménard, F. 2000, A\&A, 357, L61

Dupree, A. K., Brickhouse, N. S., Smith, G. H., \& Strader, J. 2005, ApJ, 625, L131

Edwards, S., Fischer, W., Kwan, J., Hillenbrand, L., \& Dupree, A. K. 2003, ApJ, 599, L41

Ferreira, J. 1997, A\&A, 319, 340
Ferreira, J. 2002, in Star Formation and the Physics of Young Stars, ed. J. Bouvier, \& J.-P. Zahn, EAS Publications Series, 3, 229 [arXiv: astro-ph/0311621]

Ferreira, J., \& Casse, F. 2004, ApJ, 601, L139

Ferreira, J., \& Pelletier, G. 1995, A\&A, 295, 807

Ferreira, J., Pelletier, G., \& Appl, S. 2000, MNRAS, 312, 387

Garcia, P. J. V., Cabrit, S., Ferreira, J., \& Binette, L. 2001, A\&A, 377, 609

Glassgold, A. E., Najita, J., \& Igea, J. 2004, ApJ, 615, 972

Goodson, A. P., Winglee, R. M., \& Boöhm, K.-H. 1997, ApJ, 489, 199

Grosso, N., Montmerle, T., Feigelson, E. D., \& Forbes, T. G. 2004, A\&A, 419, 653

Gullbring, E., Hartmann, L., Briceno, C., \& Calvet, N. 1998, ApJ, 492, 323

Hartigan, P., Edwards, S., \& Ghandour, L. 1995, ApJ, 452, 736

Hartigan, P., Edwards, S., \& Pierson, R. 2004, ApJ, 609, 261

Hartmann, L., Avrett, E., \& Edwards, S. 1982, ApJ, 261, 279

Hartmann, L., \& MacGregor, K. B. 1980, ApJ, 242, 260

Hayashi, M. R., Shibata, K., \& Matsumoto, R. 1996, ApJ, 468, L37

Hirose, S., Uchida, Y., Shibata, K., \& Matsumoto, R. 1997, PASJ, 49, 193

Hirth, G. A., Mundt, R., \& Solf, J. 1997, A\&AS, 126, 437

Hirth, G. A., Mundt, R., Solf, J., \& Ray, T. P. 1994, ApJ, 427, L99

Königl, A., \& Pudritz, R. E. 2000, in Protostars and Planets IV, ed. V. Mannings, A. P. Boss, \& S. S. Russell (Univ. of Arizona Press), 759

Kwan, J. 1997, ApJ, 489, 284

López-Martín, L., Cabrit, S., \& Dougados, C. 2003, A\&A, 405, L1

Lavalley, C., Cabrit, S., Dougados, C., Ferruit, P., \& Bacon, R. 1997, A\&A, 327, 671

Lavalley-Fouquet, C., Cabrit, S., \& Dougados, C. 2000, A\&A, 356, L41

Ménard, F., \& Duchêne, G. 2004, A\&A, 425, 973

Matt, S., Goodson, A. P., Winglee, R. M., \& Böhm, K.-H. 2002, ApJ, 574, 232

Matt, S., \& Pudritz, R. E. 2005, ApJ, 632, L135

Miller, K. A., \& Stone, J. M. 1997, ApJ, 489, 890

Miller, K. A., \& Stone, J. M. 2000, ApJ, 534, 398

Najita, J. R., \& Shu, F. H. 1994, ApJ, 429, 808

Ostriker, E. C., \& Shu, F. H. 1995, ApJ, 447, 813

Pesenti, N., Dougados, C., Cabrit, S., et al. 2004, A\&A, 416, L9

Ray, T. P., Mundt, R., Dyson, J. E., Falle, S. A. E. G., \& Raga, A. C. 1996, ApJ, 468, L103

Romanova, M. M., Ustyugova, G. V., Koldoba, A. V., \& Lovelace, R. V. E. 2002, ApJ, 578, 420

Sauty, C., Trussoni, E., \& Tsinganos, K. 2002, A\&A, 389, 1068

Sauty, C., \& Tsinganos, K. 1994, A\&A, 287, 893

Shang, H., Glassgold, A. E., Shu, F. H., \& Lizano, S. 2002, ApJ, 564, 853

Shang, H., Shu, F. H., \& Glassgold, A. E. 1998, ApJ, 493, L91

Shu, F., Najita, J., Ostriker, E., et al. 1994, ApJ, 429, 781

Solf, J., \& Boehm, K. H. 1993, ApJ, 410, L31

Stone, J. M., Hawley, J. F., Gammie, C. F., \& Balbus, S. A. 1996, ApJ, 463, 656

Testi, L., Bacciotti, F., Sargent, A. I., Ray, T. P., \& Eislöffel, J. 2002, A\&A, 394, L31

Trussoni, E., Tsinganos, K., \& Sauty, C. 1997, A\&A, 325, 1099

Wardle, M., \& Königl, A. 1993, ApJ, 410, 218

Woitas, J., Bacciotti, F., Ray, T. P., et al. 2005, A\&A, 432, 149 